\title{
Résister à la prison par l'autobiographie : Véra Figner et les prisons tsaristes
}

\section{Philippe Artières et Denis Dabbadie}

\section{(2) OpenEdition \\ 1 Journals}

\section{Édition électronique}

URL : http://journals.openedition.org/conflits/1562

DOI : $10.4000 /$ conflits. 1562

ISSN : $1777-5345$

Éditeur :

CCLS - Centre d'études sur les conflits lilberté et sécurité, L'Harmattan

\section{Édition imprimée}

Date de publication : 1 septembre 2004

Pagination : 15-37

ISBN : 2-7475-7583-7

ISSN : 1157-996X

Référence électronique

Philippe Artières et Denis Dabbadie, «Résister à la prison par l'autobiographie : Véra Figner et les prisons tsaristes », Cultures \& Conflits [En ligne], 55 | automne 2004, mis en ligne le 08 janvier 2010, consulté le 30 mars 2021. URL : http://journals.openedition.org/conflits/1562 ; DOI : https://doi.org/ $10.4000 /$ conflits. 1562

Ce document a été généré automatiquement le 30 mars 2021.

Creative Commons License 


\title{
Résister à la prison par l'autobiographie : Véra Figner et les prisons tsaristes ${ }^{*}$
}

\author{
Philippe Artières et Denis Dabbadie
}

Qui ne l'a pas connue la connaîtra un jour. Qui l'a connue ne l'oubliera plus ${ }^{1}$.

1 Un petit cahier sans couverture rédigé en français avec de très nombreuses corrections au crayon noir ou à l'encre, parfois des ajouts collés ou intercalés. Un second cahier d'écolier de couverture bleue, sans date, manuscrit, en français, sans correction ; un troisième cahier, celui-là de couleur gris-vert, rédigé en italien avec de nombreuses ratures. Enfin, un ensemble de feuilles volantes (vingt-sept feuillets recto), manuscrit en français d'une écriture différente, avec des corrections manuscrites du même scripteur des autres manuscrits.

2 L'auteur de ces cahiers sans date n'est autre que la populiste russe Véra Figner (1852-1942)2. Elevée à la campagne, dans la région de Kazan, Véra Figner quitte la Russie en 1872 pour faire des études de médecine. C'est là, à Zurich, qu'elle entre en contact avec le mouvement socialiste occidental, et en particulier avec la pensée de Bakounine $^{3}$. En 1873, elle participe au Congrès de l'internationale anti-autoritaire à Genève. Mettant fin à ses études, elle s'engage alors dans les luttes révolutionnaires et dès 1876 rentre en Russie. Figner devient une des plus éminentes révolutionnaires russes des années 1870-1880, chef de file du mouvement "la Volonté du Peuple», emprisonnée pendant vingt ans, de 1884 à 1904, à la forteresse de Schlüsselbourg, sur la Néva, près de Saint-Pétersbourg. Figner avait en effet joué un rôle actif dans le mouvement populiste russe responsable de l'attentat du tsar Alexandre II ; organisation politique secrète des populistes terroristes, formée en août 1879, à la suite de la scission de l'organisation populiste "Zemlia i Volia » (" Terre et Liberté »)4, la « Narodnaïa Volia » (traduit par les contemporains en français par « La Volonté du Peuple ») avait à sa tête un comité exécutif composé notamment de Jéliabov, Mikhaillov, Frolenko, Morozov, Pérovskaïa, Kviatkovski et Figner. Tout en défendant les positions du socialisme 
utopique populiste, les membres de cette organisation s'engagèrent dans la lutte politique estimant que leur tâche essentielle consistait à renverser l'autocratie et à conquérir la liberté politique. Leur programme prévoyait l'organisation d'une «représentation populaire permanente » élue au suffrage universel, la proclamation des libertés démocratiques, la remise de la terre aux paysans, des mesures pour transmettre les usines et les fabriques aux ouvriers ${ }^{5}$.

3 Figner et les membres de la Narodnaïa Volia se proposaient de transformer la société sans la participation du peuple, par le terrorisme individuel (c'est-à-dire l'assassinat de personnalités $d u$ régime tsariste), l'intimidation et la désorganisation $d u$ gouvernement. Après l'attentat contre Alexandre II du $1^{\mathrm{er}}$ mars 1881 , le gouvernement anéantit l'organisation par une répression féroce, des provocations et des exécutions capitales $^{6}$. Figner fut de ceux de la " Volonté du Peuple » qui survécurent.

Selon la Bol'chaïa Sovietskaïa Entsiclopedia rédigée sous Staline, en 1936 : «En septembre 1904, sous l'effet du mouvement révolutionnaire qui s'amplifiait, Figner fut libérée de Schlüsselbourg (...) à l'étranger, où elle séjourna jusqu'en 1915. Durant ces années, elle travailla avec des Socialistes Révolutionnaires (S.-R.), prit part au procès Azef et déploya une grande activité pour organiser des secours aux déportés et aux bagnards (...) En 1917, F. se trouvait à la tête $d u$ Comité d'aide aux bagnards et aux déportés remis en liberté». L'encyclopédie soviétique ajoutait qu'après la Révolution, Figner s'était consacrée entièrement à une activité littéraire ; ses travaux étaient jugés par les rédacteurs staliniens comme possédant des qualités artistiques certaines, mais appelaient une attitude critique sévère, car ils étaient, selon eux, bâtis sur une compréhension erronée du développement révolutionnaire russe de manière générale, de la signification historique du populisme, de la Volonté du Peuple et des Socialistes Révolutionnaires en particulier. La notice biographique s'achevait par cette remarque : «Durant les dernières années, Figner s'est mise sur la voie de l'élimination de ses vieilles conceptions relevant du populisme et de la Volonté du Peuple $»^{7}$.

Ces manuscrits intitulés "Souvenirs de la forteresse de Schlüsselbourg 》 relataient donc sa détention à la forteresse entre 1883 et 1904. Qu'étaient ces manuscrits ? Des avant-textes du récit de ses années d'enfermement qui constituait la deuxième partie "L'horloge de la vie s'est arrêtée » - des mémoires de Figner ? Ou avaient-ils une fonction particulière ? S'articulaient-ils sur une pratique politique définie? L'énigme était totale.

6 Figner avait été une extraordinaire autobiographe dont le récit principal «Le labeur scellé » publié à Moscou en 1921 et 1922 ne fut traduit en français qu'en 1973 sous le titre Mémoires d'une révolutionnaire et dans une version tronquée de 332 pages. Ces cahiers retrouvés, comme on le verra, appartenaient d'une part à un ensemble considérable et méconnu d'écrits autobiographiques de Véra Figner dont la rédaction et la publication s'étendirent de 1883 à $1932^{8}$. Les cahiers manuscrits appartenaient d'autre part à un corpus autobiographique absolument exceptionnel, celui des récits d'enfermement des militants de "la Volonté du Peuple» au sein de la forteresse de Schlüsselbourg. Autrement dit, à partir de ces quelques cahiers, c'était un vaste continent autobiographique qui se dévoilait et qui, au regard des travaux récents sur l'autobiographie dans le monde communiste ${ }^{9}$, apparait particulièrement passionnant pour étudier la fonction du discours autobiographique dans la propagande révolutionnaire. 
7 Figner fut donc épargnée par les purges staliniennes en raison de son action au début des années 1880. Comme une quarantaine de militants du mouvement populiste «la Volonté du Peuple», elle avait été emprisonnée du milieu des années 1880 aux premières années du siècle dans la forteresse de Schlüsselbourg : cette prison exclusivement réservée aux politiques appartenait à un vaste système pénitentiaire qui, en 1884, ne comptait pas moins de 792933 détenus. Un système vétuste, très dur, qui, selon un témoin de l'époque, le journaliste américain G. Kennan, comptait 884 prisons. "Il serait difficile de trouver», écrit ce témoin, «dans le monde cultivé, un autre système pénal dans lequel l'arbitraire et l'humeur personnelle jouent un aussi grand rôle $»^{10}$. Une situation critique qui s'expliquerait, selon les historiens, par l'usage des arrestations arbitraires pour inspirer la terreur dans un contexte de vague intense d'attentats terroristes ${ }^{11}$; la prison devint, alors, avec la généralisation de la détention préventive de "suspects politiques » durant des mois et des années, un outil de réponse à la violence politique des révolutionnaires ${ }^{12}$. En dépit de certaines exceptions comme la création en 1864 de maisons d'arrêt et d'asiles correctionnels pour les jeunes détenus qui sont construits, soutenus et dirigés par les «Zemstvos » (associations privées), les prisons russes sont à la fin des années 1870 dans un état de désorganisation et de vétusté extrêmes. Ce constat est corroboré par le Gouverneur général Anutchiw qui présente, en 1881, un rapport sur l'état lamentable des presque 900 prisons russes : entente des employés et des fournisseurs, tolérance de l'ivrognerie, du jeu, de l'immoralité parmi les prisonniers, châtiments corporels appliqués arbitrairement, et personnels de surveillance corrompus... Tous les observateurs reconnaissent ainsi la noirceur du tableau pénitentiaire russe. Qui plus est, à l'image du cas de la maison de détention préventive de Saint-Pétersbourg, pourtant construite entre 1873 et 1875, pour 800000 roubles, les prisons russes sont insalubres et les maladies y sont très répandues : à la maison de détention, où dès son ouverture presque $20 \%$ des prisonniers se retrouvent à l'hôpital. Le problème le plus grave auquel le pouvoir tsariste est confronté est sans conteste la question de l'autorité au sein des établissements : tous dénoncent une usurpation de l'autorité légale par les caprices des fonctionnaires; le traitement des prisonniers ne semble plus déterminé par la loi mais par le bon plaisir du procureur ou du chef de la gendarmerie. Face à cette situation, et sous l'influence des politiques menées par leurs voisins européens, la loi de 1879 prône la création d'une agence centrale spéciale chargée de la direction du régime pénitentiaire ${ }^{13}$. Malgré ces réformes les prisons tsaristes continueront à constituer des lieux de non-droit comme beaucoup d'observateurs le notent ${ }^{14}$.

8 La forteresse de Schlüsselbourg, "forteresse de la clé », construite en 1323 par un prince de Novgorod, objet de nombreuses batailles, reconquise définitivement par Pierre le Grand aux Suédois en 1702, fut très rapidement transformée en prison ${ }^{15}$. Cette forteresse devint au cours des années 1880 le symbole de l'enfermement tsariste. On la surnomma en cette fin de XIX ${ }^{\text {ème }}$ siècle "la bastille russe ", le règlement étant, même s'il varie selon les périodes, des plus rigoureux: isolement, surveillance constante, interdiction de parler, de faire du bruit, d'écrire, de se coiffer...

C'est non loin de Saint-Pétersbourg, à l'endroit le plus large de la Néva, à sa sortie du lac de Ladoga, que se trouve sur une petite île, jetée au milieu des flots, la forteresse que les Russes et les Suédois s'étaient disputés pendant plusieurs siècles. Les murs blancs de la forteresse et ses tours rondes sont d'un bel effet sur la surface sombre du fleuve... Une flèche solitaire à l'extrémité de laquelle brille une clé dorée indiquait que nous étions amenés à la forteresse de Schlüsselburg, forteresse de la clef. Avant d'avoir aperçu cette clef, aucun prisonnier ne savait en effet où on le 
conduisait. Elle semblait dire qu'une fois enfermé là, il était impossible d'en sortir. La porte ressemble à une gueule béante, et, au dessus, on voit un emblème : l'aigle à deux têtes étend ses serres d'oiseau de proie, et des lettres, inégales et à moitié effacées par le temps, forment l'inscription sinistre « ...de sa Majesté ».

Et de même que ce pauvre îlot, avec sa lande étroite de terre, se trouve loin du continent, de même l'existence de la forteresse s'écoulait loin de l'océan général de la vie.

A partir du moment où le prisonnier franchissait le seuil de la prison, le mystère et le silence l'entouraient... Nos pères et nos mères ne savaient pas où nous étions et si nous étions vivants. Nous laissions derrière les murs de la forteresse notre pays et notre action, nos amis et notre famille. Nous laissions l'humanité toute entière avec sa vie... Nous laissions même nos noms, et n'étions plus que des numéros.

Manuscrit des Souvenirs

Nous nous proposons ici de présenter les principaux résultats de l'enquête que nous avons menée sur les fonctions de l'écriture autobiographique chez Véra Figner en nous attachant au récit central de l'emprisonnement ${ }^{16}$. Il s'agit en effet d'interroger une pratique d'écriture et une pratique politique ; autrement dit, de tenter d'appréhender l'articulation de l'individuel et du collectif, de saisir une pratique intime dans son rapport à l'engagement politique. Cette enquête s'appuie sur le dépouillement des archives Figner à RGALI ${ }^{17}$, les différentes éditions des mémoires de Véra Figner en russe, en anglais (1927) et en français (1930 et 1973), les écrits d'autres prisonniers de la Forteresse de Schlüsselbourg ainsi que la presse (La Tribune russe, notamment) et les revues de l'époque (Byloïé) ${ }^{18}$.

\section{7-1904 : en prison, écrire/survivre}

La plus ancienne strate du corpus autobiographique de Véra Figner que nous ayons à ce jour retrouvé date de 1887, c'est-à-dire des années de prison : il s'agit majoritairement de poésies dont les manuscrits originaux ont été détruits. Ces poèmes, comme celui ciaprès, rédigé le 13 décembre 1887, intitulé «Tac-tac » et dédié à Pankratov, évoque l'enfermement de manière très allusive :

C'est assez, voisin, d'étudier !

Quelles sciences n'existe-t-il pas?!

Si à toutes on s'attaque

Mon ami, la vie n'y suffira pas !..

Sors du temple des sciences !...

Et réponds aux coups que je frappe...

Tac-tac!

11 On peut se demander pourquoi cette prise d'écriture n'intervient qu'en 1887. Véra Figner est transférée à la forteresse de Schlüsselbourg dès 1884. Pendant les trois premières années, elle demeure dans un isolement total ; elle ne peut ni lire, ni voir ses camarades ; ils communiquent par un système de biftons et de coups contre le mur. Trois années passent au cours desquelles plusieurs militants deviennent fous, se suicident ou meurent de maladie. Véra tient bon. En 1887, un adoucissement du régime d'incarcération intervient : du papier et des crayons sont donnés aux détenus.

On soulignera que les variations du règlement sont nombreuses au cours de ces vingt ans et qu'alternent ainsi des périodes de relative clémence (accès à des revues françaises et anglaises, entrée de nombreux livres, autorisation de promenades collectives, d'entretien d'un potager, etc.) et des périodes d'extrême sévérité 
(enfermement en cachot, violences, multiplication des fouilles...). Ces dernières arrêtent parfois net les pratiques d'écriture et réduisent à néant les manuscrits produits puisque aucun texte, à notre connaissance, ne sortit illégalement de la forteresse quelle que soit la libéralité du régime.

Si les premiers écrits autobiographiques de Figner sont des poèmes et non un récit autobiographique ou un journal intime, c'est sans doute d'une part que le récit autobiographique est associé chez elle à l'aveu - au moment de son arrestation, elle doit faire son autobiographie contrainte pour la police (celle-ci sera publiée en 1917 dans la revue Bylö̈́) - et de même que le journal intime, il est dangereux, car largement suspect aux yeux de ses gardiens qui inspectent sa cellule au moins une fois par semaine, comme l'écrit un autre prisonnier, Aschenbrenner, dans ses mémoires : « Chacun des détenus songeait à tenir un journal, mais cela était malheureusement impossible, car on nous fouillait avec soin, à peu près jusqu'en 1896, et même ensuite on farfouillait dans nos papiers, et nous ne souhaitions pas nous épancher sous les yeux de nos persécuteurs. Des journaux auraient montré un tableau vivant de la vie tragique dans laquelle périrent tant de personnages, ou, du moins, auraient donné des clichés photographiques de cette vie. Les présentes notes sont seulement un pâle schéma, dépourvu de vivacité, de vie et de pathétique " ${ }^{19}$.

Pratique dangereuse, le journal était donc peu pratiqué, bien que Véra Figner rapporte qu'un autre détenu, l'ouvrier Martynov, parvint à en tenir néanmoins un et qu'il réussit grâce à un bienveillant gardien à le faire parvenir à la Police ; il y faisait une description des fouilles dont les détenus étaient l'objet ; à partir de ce moment-là ces fouilles cessèrent brusquement.

Quant à la correspondance avec l'extérieur, elle n'est autorisée qu'à partir de 1896, soit treize ans après le début de l'incarcération, et de manière très limitée, deux fois par an seulement. Ces lettres sont l'objet de la plus vive surveillance : aucune mention ne doit être faite des conditions de détention et de la vie en prison. Le discours autobiographique en est donc banni. Qui plus est, comme Véra Figner l'explique à sa mère et à ses sœurs dans une lettre conservée du 2 mars 1900, l'emprisonnement a altéré son rapport à l'écriture :

(...) une incapacité complète à faire entrer quoi que ce soit dans la forme et dans le cadre d'une lettre. J'ai perdu l'habitude d'exposer quoi que ce soit par écrit ; j'ai perdu l'habitude de ce moyen de communiquer avec vous, et, en vérité, la télépathie m'irait maintenant mieux que tout. La pensée et la parole sont en quelque sorte séparées chez moi et ne veulent pas s'unir : à peine je commence à écrire, un trouble mécontentement surgit (...) et, impuissante, je baisse le bras. N'est-ce pas là les affres du verbe qu'éprouvent tant le haut du pavé de l'humanité en la personne des poètes, des artistes des belles lettres que le bas du pavé de la culture humaine, les gens tout à fait simples et peu instruits dont la pensée et le sentiment grouillent dans le cerveau, et les mots leur manquent pour les exprimer. Ou bien, peut-être, est-ce là une maladie, résultat de l'activité du microbe pénitentiaire. En effet, cela ne m'arrivait pas avant. Vous vous souvenez, ma petite maman, Olga disait même que j'avais l'art d'écrire des lettres... de même qu'il y a le pouvoir de la terre formulé par Gleb Ouspenski, il y a le pouvoir de la prison, quand même une occupation qui, apparemment, est si sujette au bon plaisir et exige la présence d'un certain état d'esprit, comme la lecture, même elle devient quelque chose de forcé. Par exemple, nous répartissons entre nous les lectures dans un certain ordre de succession... Déroger à cet ordre signifie y déroger pour tous, tout embrouiller...20

16 La poésie devint ainsi la pratique d'écriture de soi la plus commune car la moins risquée, puis, le régime s'adoucissant, des romans furent rédigés. V. Figner écrivit de 
" charmants petits contes et de poétiques souvenirs sur sa jeunesse ", selon la formule de l'un de ses co-détenus, et des traductions, en particulier celle de Kim de Kipling ; elle apprit en outre l'italien. Autrement dit, la forteresse se transforma en un vaste atelier d'écriture.

De ces plusieurs centaines de pages manuscrites produites dans cet atelier, il ne reste malheureusement qu'un tas de cendres. En effet, quelques jours avant sa libération en 1904, Figner détruit l'ensemble de ses écrits.

Véra Figner sort donc de la forteresse sans un manuscrit relatif à son incarcération ; mais elle a pris soin d'apprendre par cœur l'ensemble des poèmes qu'elle et ses camarades ont rédigés, et elle les publie dès 1906. Elle précise dans sa préface la forte valeur autobiographique de ces poèmes : "J'avoue que j'ai une attitude négative envers l'intérêt artistique de mes vers. Leur véritable place serait, me semble-t-il, dans mes souvenirs de la forteresse de Schlüsselbourg... et hors des mémoires, quel est leur poids spécifique?».

19 Les archives de RGALI conservent le manuscrit de ces poèmes sortis de mémoire de la prison. Il s'agit d'un petit carnet noir de cent quatre pages, comprenant quarante-neuf poèmes et intitulé "Cahiers de poèmes écrits par des prisonniers de la forteresse de Schlüsselbourg ayant appartenu à VF ». Dans la deuxième partie de ses mémoires publiées en 1922, Véra reprend quelques-uns de ses poèmes dans le chapitre qu'elle consacre à l'arrivée du papier.

20 On retrouve également dans cette deuxième partie quelques lettres qu'elle avait envoyées alors à sa famille, notamment sa dernière lettre publiée dans le chapitre « $\mathrm{A}$ la veille » qu'elle retrouva à sa sortie, après deux années de déportation dans la province d'Arkhanguelsk, en allant chez sa sœur. Figner publiera enfin en 1929 et 1932, l'ensemble de sa correspondance sous le titre, Lettres de Schlüsselbourg (soixante-dix pages) dans ses œuvres complètes en sept volumes.

21 A sa libération, Véra Figner, en même temps qu'elle publie les poésies de Schlüsselbourg, se livre à une curieuse entreprise d'écriture.

\section{4-1906 : en exil intérieur, écrire pour se souvenir}

Véra Figner quitte Schlüsselbourg le 29 septembre $1904^{21}$ mais ne peut emporter toutes ses archives : "Dans les cahiers, il ne doit rien y avoir sur Schlüsselbourg »; elle brûle tout ce qui s'y rapporte. Elle s'exclamera dans Le Labeur scellé : «Adieu, chères lignes! Un an ou deux passeront, et je ne serai plus en état de vous reproduire pour moi avec exactitude. Il ne restera qu'une faible trace, un sentiment général de reconnaissance envers ceux qui vous ont écrites. Ce que je perds maintenant avec vous, je le perds sans retour et pour toujours ».

23 Il convient de garder ce profond traumatisme toujours à l'esprit, qui est non pas un double « éclairage », mais une double " offuscation », confiscation : de Véra Nicoläievna en tant que personne et de l'ensemble de ses écrits.Ajoutons que Figner part alors en exil en province et qu'elle ne pourra pendant de longs mois retrouver ses proches. Un peu plus d'un an passa effectivement, et 1906 vit Figner publier une plaquette de vers et une biographie de Lioudmila Wolkenstein. C'est au lendemain de la mort prématurée de celle-ci que Figner rend hommage à son ex-codétenue qui avait été la première à écrire ses souvenirs de Schlüsselbourg, et ce dès sa libération en $1896^{22}$. Daté du 25 février 1906 et publié dans le numéro de mars de la revue Byloié, le texte sera repris mot pour 
mot dans Le Labeur scellé, avec pour seul ajout un «comme moi » qui est caractéristique de la progressive reconquête d'une identité.

Cette même année 1906, précisera Figner dans la préface du tome 4 de ses œuvres complètes, "j'ai écrit les essais de six camarades proches pour la Galerie des prisonniers de Schlüsselbourg... elle avait pour but d'augmenter les fonds du 'Comité de Schlüsselbourg' fondé d'après mon idée par P. Iakoubovitch, en vue de la sortie possible des neuf membres de la Volonté du Peuple qui y étaient restés après moi ».

Ainsi, sortant de l'épreuve de vingt années d'emprisonnement, Figner opère en somme un premier travail ; elle se constitue en mémorialiste de la Forteresse. Elle cherche par ce travail d'écriture à contrer après coup ce qui était au cœur du système d'emprisonnement de Schlüsselbourg : l'oubli. Au premier chef, ce travail de mémoire passe par la rédaction d'un ensemble de portraits de ses camarades décédés en détention. Le tsar voulait gommer de la mémoire collective "la Volonté du peuple", Figner ressuscite ses membres par l'écrit. Elle cherche ainsi à leur redonner un visage, une voix dont la prison tsariste les a privés. La pratique de l'écriture a ici certes une fonction personnelle - il s'agit de se retrouver une identité déniée pendant vingt ans -, une fonction pratique - financer l'organisation - mais également une visée politique : révéler ce qui a été tu et dont seule Figner est dépositaire. Elle se pense ainsi en dernier témoin ayant un devoir politique de noter noir sur blanc les dernières années de ses camarades disparus.

\section{7-1913 : en Europe, écrire et renouer avec le militantisme}

$26 \mathrm{Au}$ terme de cette entreprise d'écriture biographique des prisonniers de Schlüsselbourg, Figner n'amorce pas, comme on aurait pu le croire, la rédaction de ses mémoires, ou tout au moins, pas directement. Autorisée à quitter la Russie, elle va en Angleterre, Italie, Finlande, Suisse et en France - à Paris puis dans le Sud du pays - et s'engage dans la défense des prisonniers russes incarcérés. Son action vise à dénoncer les conditions de la répression politique à l'œuvre en Russie : « depuis 1905, la Russie étant devenue une monarchie constitutionnelle, nous voyons le pays se couvrir de dizaines de prisons où l'on châtie, avec une cruauté inouïe, les prisonniers politiques, tous ceux que l'œil de la police a notés pendant la révolution comme des partisans de la liberté. De la Baltique à Vladivostok, de l'Oural à la Mer Noire, nous trouvons une série de chambres de torture, renfermant des milliers de condamnés politiques, dix à onze mille prisonniers ${ }^{23}$.

A la suite de l'envoi d'une lettre d'une déportée aux mines d'Algatchi, qui a transité par sa sœur, Figner décide de fonder à Paris un Comité de secours aux prisonniers russes pour recueillir des fonds, comme elle l'explique dans la préface à la brochure qu'elle publie à Grenoble, Les Prisons russes, en 1913 : «Fidèle à son idéal, pleine d'énergie que les longues années en prison ne surent briser, d'une modestie surprenante, elle déteste la phrase et évite les expressions du sentiment et d'admiration que lui vouent ceux qui ont le bonheur de la connaître. Libre maintenant depuis plusieurs années, elle continue à agir, c'est pour venir en aide aux prisonniers politiques de la jeune génération qu'elle travaille; une caisse de secours aux condamnés aux travaux forcés fut fondée par elle en 1910 à Paris ".

C'est dans ce cadre militant que Figner commence à écrire ses souvenirs ; les manuscrits conservés à RGALI datent de cette période. Ils témoignent de cette 
entreprise autobiographique au service de la lutte contemporaine. La rédaction de ses mémoires - en 1910 - a une fonction militante évidente : il s'agit, à partir de son cas, de témoigner pour les prisonniers alors dans les geôles russes. Les récits autobiographiques inédits, s'ils serviront comme on le verra à la rédaction ultérieure en 1921 - des mémoires, sont d'abord des conférences prononcées en Europe (Italie, Suisse, Angleterre, Belgique, France) ${ }^{24}$.

On dispose donc dans les archives de plusieurs versions d'un même texte, chaque version répondant à des circonstances particulières. Bien que non datées, on peut, par leur examen, conclure que la plus ancienne version est le cahier sans couverture, rédigé en françaisseulement au recto (quarante sept folio), à l'encre violette, avec de très nombreuses corrections au crayon noir ou à l'encre, et parfois des ajouts collés ou intercalés avec page recto verso d'un texte publié avec correction et ajouts manuscrits.

Ce manuscrit est, selon nous, la toute première mouture de l'autobiographie de Figner, rédigée vers 1910 lors de son séjour en Suisse. Il s'agit d'un manuscrit de travail ou d'une première rédaction émaillée de corrections visant à une réduction : l'ensemble de celles-ci vont dans ce sens.

31 Le texte commun aux quatre manuscrits comprend plusieurs parties. Il s'ouvre non sur la naissance et l'enfance de Véra, mais sur un autre récit de naissance, celui d'une militante ; d'abord philanthrope, Figner, de retour en Russie après des études en Suisse, est révoltée par la situation dans les campagnes ; elle décide de se joindre aux mouvements révolutionnaires et à la lutte armée. Après ce préambule, dans une courte introduction, Figner, sans entrer dans le détail, rappelle les motifs de l'action de «la Volonté du Peuple ", puis narre les circonstances qui ont conduit à son arrestation. Suit un long développement qui s'organise en six points : 1 . Présentation générale de la prison (isolement, alimentation, cellule) ; 2. Le cachot ; 3. En promenade avec M. Wolkenstein ; 4. La correspondance (avec l'épisode de l'oiseau) ; 5. Les épaulettes ; 6. La libération et l'exil. Le récit s'achève sur une conclusion et un appel à la mobilisation.

Or, les épisodes du cachot, des épaulettes, de la correspondance, ainsi que de la rencontre avec L. Wolkenstein constituent l'avant-texte des chapitres des mémoires, comme l'exemple du cachot le montre puisqu'on y retrouve non seulement un récit construit avec les mêmes éléments, mais surtout la reprise de formules semblables.

"Moi-même, je fus une fois mise au cachot et voici comment : au cours de ma cinquième année de prison, un de mes camarades, vers 9 heures du soir, se mit à frapper au mur qui nous séparait afin d'attirer mon attention. Mais avant qu'il eût adressé sa première phrase, j'entendis qu'on ouvrait la porte de sa cellule et qu'on l'emmenait : cela signifiait le cachot. Je savais qu'il n'en était revenu que quelques jours auparavant ; qu'on l'y avait frappé, mis à la camisole de force et jeté à terre. Je m'imaginais quels nouveaux outrages, il allait endurer... Je me mis à frapper à la porte : le visage du directeur parut. «Il est injuste de ne punir qu'une personne lorsque deux ont parlé, mettez moi aussi au cachot », lui dis-je.

« Fort bien! » répondit avec colère le directeur, et il ouvrit ma porte. Alors, je vis pour la première fois l'intérieur de notre prison la nuit. Je vis quarante lourdes portes gris foncé : quarante entrées de tombes ; et derrière chacune, un ami, un camarade captif.

De distance en distance, une petite veilleuse, accrochée au mur, jetait une lueur avare, ce qui donnait encore plus l'aspect d'une crypte à l'intérieur de la prison.

Je ne pus m'empêcher de chuchoter : "On me mène au cachot ». La sonorité du bâtiment était telle qu'au fond de leurs cellules tous mes camarades entendirent ces mots, et répondirent aussitôt : «Qu'on nous mette tous au cachot !»... Et la prison retentit du bruit des coups dont ils ébranlaient les portes... 
Le directeur, hors de lui, descendit l'escalier et quand nous fûmes dans la cour, il brandit devant mon visage son poing qui tenait la lourde clef de la prison et me dit : «Recommencez donc ici vos plaisanteries et vous allez voir!" Cet homme me faisait peur : son geste était trop expressif pour n'être pas compris et la pensée me traversa l'esprit que s'il portait la main sur moi, je me tuerai... Dominant mon agitation, je répondis d'un ton calme : «Ce n'est pas dans ce dessein que je vais au cachot ».

La seconde version en français, non datée, fut écrite sur un cahier d'écolier de couverture bleue à l'encre noire sans correction. Soit trente folios recto-verso et un recto et quelques pages blanches qui correspondent à la mise au propre de la première version sans autres ajouts. La troisième version est en italien, sur un cahier d'écolier de couleur gris-vert. Si ce manuscrit comporte des ratures, celles-ci sont seulement relatives aux difficultés de traduction (Figner ayant appris l'italien en détention), il est la traduction mot à mot du texte original. Enfin, le quatrième manuscrit en français, sur feuilles libres (vingt-sept feuillets recto) d'une écriture différente avec correction de Véra Figner à l'encre noire, est moins un manuscrit qu'une copie avec correction autographe de Figner ; d'après quelques indications, ce texte semble destiné à un auditoire anglais, alors que les trois précédents étaient destinés à un public suisse et italien. Les souvenirs sont ici largement remaniés et la fin du texte est totalement différente. Le récit de l'emprisonnement à Schlüsselbourg est raccourci. Le récit de l'Hirondelle - par lequel Figner narre la relation qu'elle entretint dans sa cellule avec un oiseau - disparaitt par exemple dans cette version (p. 7) ; de même, le récit du cachot est plus ramassé, tandis que le dernier épisode concernant le renforcement du régime pénitentiaire - les épaulettes - est tout simplement supprimé. La fin du récit est également différente; Figner insiste sur l'actualité de la prison en Russie : à la place de "A l'heure actuelle, le tableau radieux s'est obscurci ", on trouve "Aujourd'hui cette vision est assombrie : de terribles souffrances sont redevenues le sort de notre pays... » et le manuscrit s'achève sur une lettre d'une exilée introduite par ces mots de Figner: "Pour vous donner une idée de la condition misérable et indigente où vivent des dizaines de milliers de jeunes gens, je vais vous lire une lettre d'une jeune institutrice exilée dans le district de Tobelsta en Sibérie ». Suit la lettre :

Je gagne ma vie en faisant la lessive et le ménage des paysans les moins pauvres du village ; et de la sorte, je parviens à gagner 2 à 3 shillings par semaine; je paie 2 shillings par semaine à la veuve Matriona pour un coin dans une cuisine où je dors. $\mathrm{Au}$ début pour toute nourriture, j'avais les restes des repas de mes amis paysans. Chaque fois que j'avalais un morceau de pain noir et d'orge, je me disais que j'étais une charge pour une famille pauvre et nombreuse. Mais malgré cette idée, le cœur douloureux, j'avalais les croûtes de pains rassis - n'ayant aucun autre moyen pour ne pas mourir de faim - Parfois honteuse, je refusais ce repas misérable. Je me jetais sur mon lit, dans un coin et je me cachais le visage pour essayer de dormir tandis que mon imagination enfiévrée me représentait des scènes de confort et d'abondance. Mais les mirages ne pouvaient apaiser ma faim. A bout de forces, il m'arriva de mendier quelques croûtes à une mendiante qui vivait elle-même de la charité des paysans ; et je les dévorai avidement. La faim peut être satisfaite un moment, mais il n'y a aucun espoir de changement pour l'avenir. En mars, un exilé politique Govorov est mort ici de faim, il a laissé une lettre disant qu'il n'a rien mangé depuis une semaine. Son image me hante incessamment.

Il est vraisemblable que Véra Figner ait rédigé cette autobiographie lors d'un déplacement en Angleterre, dans le cadre d'une campagne de soutien aux prisonniers russes. Il faut ici souligner que ces conférences autobiographiques de Figner assemblent 
un double matériau : d'une part, ses souvenirs de Schlüsselbourg, et d'autre part les récits autobiographiques reçus des prisonniers alors incarcérés ou déportés

C'est ce même usage des écrits personnels qui prévaut dans la publication par Véra Figner de la brochure Les Prisons russes à Lausanne, en 1911 : "Le chef du service pénitentiaire sous l'égide duquel s'accomplissent les actes de barbarie, nous assure que toutes les horreurs sont inventées, que les prisonniers mentent. Mais on ne ment pas dans la correspondance intime, on ne raconte pas l'humiliation subie, si cette humiliation n'est pas réelle. Que le lecteur juge par lui-même, d'après les documents qu'il trouvera plus bas, qu'il dise où est la vérité » (p. 16). On retrouve dans cette brochure la trace des souvenirs, mais leur caractère personnel est totalement gommé au profit d'un exposé de la situation contemporaine.

Ecrire la biographie des autres, et notamment de Wolkenstein, c'était se souvenir et faire le deuil des camarades disparus et, en même temps, commencer à écrire ses propres mémoires, comme Figner le note en 1932 lors de la publication en livre de ses biographies : "le lecteur trouvera ici une répétition inévitable d'une partie de ce qui a trouvé place dans le second volume de mes œuvres ». Militer en écrivant, c'est tenter de retrouver une place dans le mouvement révolutionnaire contemporain. Car Figner, à partir de son expérience d'enfermement construit un récit qui articule son passé au présent des détenus de la fin de la première décennie du siècle. Ses textes entremêlent son récit personnel avec le témoignage d'autres, constituant le discours du prisonnier victime du tsarisme. Ce prisonnier n'a pas de nom, il est une sorte de figure emblématique dont Figner témoigne. Sans doute y a-t-il dans cet engagement un souci de ne pas oublier le passé tout en se projetant dans un avenir, celui de la disparition des prisons et des bagnes tsaristes. C'est cette dynamique qui est à l'œuvre lors de son séjour en Suisse et en France. User de son talent d'écrivaine et de son expérience d'enfermement pour faire entendre la voix des prisonniers. Enfin, en centrant son discours sur l'arbitraire de la détention en Russie et les conditions d'enfermement, l'objectif de Figner est de mobiliser le plus grand nombre contre le tsarisme en usant d'un thème sensible. L'écriture de la prison a donc aussi une visée stratégique.

Bien que Figner ait conservé toute sa vie la direction des Comités de secours aux prisonniers, il semble qu'à partir de 1913, elle prenne du recul par rapport aux événements contemporains et que c'est à ce moment-là qu'elle commence à écrire véritablement ses mémoires pour eux-mêmes, dans une vocation littéraire qu'elle ne quittera plus jusqu'à sa mort en 1942. Ainsi, dans la présentation à la seconde édition des Prisons russes, le préfacier qui est probablement Véra Figner écrit : «En ce moment Mme Figner écrit ses mémoires »...

\section{3-1922 : écrire pour l'histoire}

« Ecrivez, me disait, lors de notre rencontre à l'étranger, la grande tragédienne Eleonora Duse. Ecrivez : vous devez écrire ; votre expérience ne doit pas disparaitre ». Et c'est à l'étranger que Figner se replonge dans ses années schlüsselbourgeoises, principalement en Suisse, à Clarens, sur les bords du lac de Genève...

Dans sa préface à la première édition, Figner livre certaines précisions fort précieuses sur la genèse de son texte, même si elle les interrompt tout à coup par un déroutant : "Il serait ennuyeux d'énumérer les moments et les lieux où fut écrit le reste. J'avais couché sur le 
papier ce chapitre ['Le premier jour', texte inaugural, donc, à tous points de vue] en 1910 [une note indique même : Le 14 décembre 1910]... C'est seulement deux ans plus tard, au printemps 1913, que je revins à la description ultérieure de mes impressions de la forteresse de Schlüsselbourg ".

Véra Figner revient en Russie en 1915, grâce à son frère, le ténor Nicolaï Figner, célèbre chanteur des Théâtres Impériaux. Cela ne l'empêche pas d'être fouillée et arrêtée à la frontière. Prudente, elle avait laissé ses manuscrits en Suisse... « A partir de 1916/17, j'ai vécu de mon travail, insérant dans des revues certains chapitres de mes souvenirs ", nous apprend-elle dans l'autobiographie (écrite en 1926) publiée dans le Dictionnaire encyclopédique Granat.

41 C'est en 1916 que ce qu'elle nomme elle-même «les meilleurs chapitres de mon livre » est révélé d'abord à quelques privilégiés, puis à un plus large public : «V. Lébédéva... réunit dans son salon une dizaine d'hommes de lettres. Il y avait là Véréssaievv, Valéri Brioussov, Alexis Tolstoï, Bounine... Je devais leur lire quelques chapitres de ce que j'avais écrit durant l'été. Je choisis 'Dix jours', 'L'éveil' et encore quelque chose... Véréssaïev, Bounine et d'autres membres de la maison d'édition 'Les écrivains moscovites' insistèrent énergiquement afin que je donne ces chapitres pour leur recueil, où ils apparurent pour la première fois, et ensuite ils figurèrent dans le second volume du Labeur scellé ».

L'amnistie politique consécutive à la révolution de 1917 la propulse Présidente du Comité d'aide aux bagnards et aux déportés libérés : "Passèrent par le Comité plus de 4 mille personnes [105 mille roubles furent envoyés par moi en Suisse pour faire sortir des émigrés ; parmi eux il y eut... Lénine et ses camarades]». Parallèlement, elle récupère ses dépositions de 1883, qui sont publiées dans la revue Byloïé...

En 1920, son recueil Les Prisonniers de Schlüsselbourg est son premier livre édité à Moscou. Il est suivi, en 1921, du premier tome du Labeur scellé, consacré aux souvenirs antérieurs à Schlüsselbourg. Trois jours plus tard, elle reçoit les manuscrits laissés à l'étranger en 1915. Le deuxième tome, qui décrit sa détention, paraît en 1922.

Véra Figner collabore en même temps à la revue Bagne et déportation (qui ne sera suspendue qu'en 1935). Comment mesurer le grand écart qui s'imposait ainsi à elle entre passé et présent?

Ainsi, alors que la Révolution est en route en Russie, Figner rédige ses souvenirs; il semble que ce soit l'urgence qui la pousse dans la rédaction de son autobiographie. Mais il est probable que si Figner se lance à corps perdu dans cette entreprise, c'est qu'elle considère que son action doit désormais passer par l'écriture; c'est par l'écriture qu'elle participe à l'histoire ; la Révolution qui advient a une histoire et il lui appartient de la restituer. Elle en est une des actrices et son devoir pour les générations à venir est de peindre les événements qui ont précédé le renversement du tsar et le portrait de ceux qui ont résisté au tsarisme.

\section{5-1926 : écrire/résister}

Véra Figner participe au tome 40 de l'encyclopédie Granat consacré à un thème brûlant : le socialisme. Elle réunit et présente quarante-quatre autobiographies dont la sienne. Elle conclut : «Le facteur fondamental de ma vie fut Schlüsselbourg. Il m'a pris 20 années de ma vie et, me coupant pour une période si longue, ininterrompue, de son cours général, m'a rejetée dans d'autres générations, dans un milieu broyé par la progression du développement 
économique et social. Dépasser l'évolution réalisée, se fondre avec tout ce qui avait changé, était nouveau, s'est avéré impossible. Et cela a fait mon malheur ».

On admirera ici, dans un article d'encyclopédie, la constance avec laquelle Véra Figner répète sa préface, écrite en 1924, pour Après Schlüsselbourg : «Est-il incompréhensible que, après un isolement de vingt-deux années, jetée dans le cours de la vie commune, je n'aie pas pu me fondre avec lui,j'ai été incapable de trouver ma place dans la vie?". Les années 1920 sont donc pour Figner celles de la désillusion; elle qui croyait participer du grand mouvement révolutionnaire est alors isolée ; elle le reste jusqu'à la fin de sa vie. L'incarcération à Schlüsselbourg l'a coupée de l'action, mais surtout c'est la défaite de ses idées, celles de " la Volonté du Peuple », face à celles des bolcheviks qui a contribué à cet isolement; l'écriture a dès lors une fonction de survie ; continuer à écrire pour lutter contre la solitude et se reconstituer dans l'écrit une communauté, la communauté de ses camarades morts à Schlüsselbourg.

L'originalité du cas Figner est qu'il fonctionne à l'inverse des autres; la «Vénus de la Révolution» ne commence pas par écrire ses mémoires pour ensuite utiliser cette parole autobiographique dans un cadre militant: c'est son engagement politique qui motive une pratique d'écriture personnelle. Tout se passe comme si avec Véra Figner le " je » passait toujours par un "nous », comme si, en définitive, on était en présence d'une impossible autobiographie à la première personne.

\section{ANNEXES}

\section{Annexe - Eléments biographiques}

1852 : Naissance de Véra Figner le 25 juin (7 juillet).

1855 : Mort de Nicolas I ${ }^{\text {er }}$. Alexandre II empereur.

1861 : Abolition du servage.

1881 : Alexandre II périt à la suite d'un attentat. Pendaison de Sofia Pérovskaïa.

Février 1883 : Arrestation de Véra Figner. Dépositions.

1884 : Procès des Quatorze. Transfert à la forteresse de Schlüsselbourg.

1887 : Premiers poèmes.

1896 : Nicolas II empereur.

1897 : Première lettre de Schlüsselbourg (2 mars).

1900 : Publication des souvenirs de Lioudmila Wolkenstein (écrits en 1896).

29 septembre 1904 : Véra Figner sort de la forteresse de Schlüsselbourg.

1905 : « Dimanche sanglant ». 
1906 : Publie une plaquette de ses Poèmes.

Ecrit une biographie de L. Wolkenstein, puis celles de six autres codétenus.

1906-07 : Parution des souvenirs de M. Aschenbrenne

et des Notes de M. Novorousski.

1907 : Ecrit quatre nouvelles biographies.

Edition de la Galerie des prisonniers de Schlüsselbourg.

Fin janvier 1910 : Création du Comité parisien d'aide aux bagnards politiques russes. Tournée de conférences.

1911 : Publie à Lausanne une brochure sur Les Prisons russes.

Printemps 1913: rédige de nouveaux chapitres de ses souvenirs

de Schlüsselbourg. A Grenoble, nouvelle édition, augmentée

des Prisons russes.

1915 : Retour en Russie. Véra Figner est arrêtée.

1916 : Ecrit, près d'Oriol, « les meilleurs chapitres » du Labeur scellé et les premiers chapitres d'Après Schlüsselbourg.

Lit à Moscou, devant, entre autres, V. Brioussov, I. Bounine,

Alexis Tolstoï, « Dix jours » et « L'éveil ».

1917 : Parution dans Byloïé (août, sept., oct.) des dépositions de 1883.

Février 1921 : rédige le deuxième chapitre de ses souvenirs

sur Schlüsselbourg («Les premières années »).

Décembre 1921 : parution du tome 1 du Labeur scellé

(souvenirs antérieurs à Schlüsselbourg).Trois jours après, elle reçoit les manuscrits laissés à l'étranger en 1915.

Juin 1922 : rédige « Les lettres brûlées ", chap. XXXII de ses souvenirs de Schlüsselbourg. Parution du tome 2 du Labeur scellé

(souvenirs de Schlüsselbourg).

1923 : Ecrit les vingt premiers chapitres d'Après Schlüsselbourg.

1924 : Parution des ch. I-XIV d'Après Schlüsselbourg. Mort de Lénine.

1925-26 : Réunit et présente quarante-quatre autobiographies,

dont la sienne, dans l'encyclopédie Granat.

1927 : Ses souvenirs paraissent en anglais à New York.

1928 : Edition allemande publiée à Berlin.

1929 : Euvres complètes en six volumes. Trotski expulsé d'URSS

1930 : Victor Serge publie en français le premier tome

du Labeur scellé, sous le titre Mémoires d'une révolutionnaire. 
1932 : Euvres complètes en sept volumes.

1935 : Suspension de la revue Bagne et déportation, à laquelle elle collaborait depuis 1921.

1936-37 : Procès de Moscou

1941 : L'armée allemande envahit l'URSS.

1942 : Meurt le 15 juin.

1964 : Réédition en URSS du Labeur scellé.

1973 : Nouvelle édition en France (Denoël) des Mémoires d'une révolutionnaire, qui inclut les souvenirs de Schlüsselbourg.

\section{NOTES}

*. Cette enquête s'inscrit dans un PICS du CNRS avec la Russie dirigé par Catherine Viollet (ITEM/ CNRS).

1. Proverbe russe sur la prison, cité par Jacques Rossi, Manuel du Goulag: dictionnaire historique, Paris, Cherche Midi, 1997, page 213.

2. Il n'existe aucune biographie de Véra Figner, on peut néanmoins consulter : Goldsmith M., Cinq femmes contre le monde [Flora Tristan, Louise Michel, Véra Figner, Emma Goldman, Rosa Luxembourg] traduit de l'anglais par Susan Sandry, Paris, Gallimard, 1937. Voir aussi la remarquable présentation de Fernand Rude de l'édition française des Mémoires d'une révolutionnaire, Paris, Denoël/Gonthier, 1973, pp. 9-21. Certains éléments biographiques figurent en annexe de cette contribution, page 35 et s.

3. Bakounine avait fondé à Zurich au cours de l'été 1872 une section slave de l'Association internationale des Travailleurs.

4. Fondée en 1876 par de jeunes étudiants russes, l'organisation Terre et Liberté, était fortement inspirée des thèses de Bakounine, notamment de son catéchisme d'un révolutionnaire (1869).

5. Véra Figner qualifie ainsi dans ses mémoires le but de «la Volonté du Peuple»: «La transmission de la terre, principal moyen de production, à la communauté paysanne, et le remplacement de la souveraineté absolue d'un seul par la souveraineté absolue du peuple, c'est-à-dire l'établissement d'un régime dans lequel la volonté librement exprimée du peuple eût été le régulateur unique de la vie sociale». 6. A plusieurs reprises, certains cherchèrent à ressusciter la « Narodnaïa Volia » mais ces tentatives furent vaines. Ainsi, en 1886 surgit un groupe dirigé par le frère de Lénine, A. Oulianov et $P$. Cbévyrév qui s'en inspira. Après une tentative avortée d'organiser un attentat contre Alexandre III en 1887, le groupe fut découvert et ses participants actifs exécutés. Après la Révolution de 1917, ceux qu'on appelait les narodniki furent respectés pour leurs actions bien que ne s'inscrivant pas dans la même lignée que les bolcheviks. Lors des purges staliniennes des années 1930, à l'image de Véra Figner, considérée comme la Venus de la révolution, ils ne furent pas inquiétés.

7. Dans les Mémoires d'un révolutionnaire (1901-1941), Paris, 1951, Victor Serge note, page 302, que l'une des dernières représentantes des narodniki à la fin de sa vie se considérait « comme le vivant symbole des générations révolutionnaires passées, qui furent celles du pur sacrifice... Frêle, dure et droite, exigeante envers elle-même autant qu'avec les autres ». Bien qu'incarnant un symbole, Figner fut durant les dernières années de son existence mise en liberté surveillée. La revue Bagne et déportation qu'elle dirigeait cessa de paraître; nombre de ses amis furent victimes de la répression stalinienne. Figner n'hésita pas à écrire au Comité exécutif, à Kalinine et à Staline luimême pour protester.

8. Voir les détails de ce corpus en annexe dans les repères biographiques. 
9. Voir sur ce point les deux ouvrages collectifs suivants : Studer B., Unfried B. et I. Herrmann (éd.), Parler de soi sous Staline. La Construction identitaire dans le communisme des années trente, Paris, MSH, Colloquium, 2002 ; Pennetier Cl., Pudal B. (éd.), Autobiographies, autocritiques, aveux dans le monde communiste, Paris, Belin, 2002.

10. Kennan G., Les prisonniers politiques en Russie, Genève, Librairie Stapelmohr, 1896.

11. C'est la thèse de M. Gernet in History of the Prisons in Imperial Russia, 5 volumes, Moscou, 1960-1963.

12. Artières Ph., «Une prison tsariste : le cas de la forteresse de Schlüsselbourg » in Artières Ph. et P. Lascoumes (dir.), Gouverner, enfermer, Paris, Presses de sciences po, 2004, pp. 257-267.

13. Cette nouvelle politique est élaborée avec le concours du spécialiste belge des prisons M. Stevens. Elle est plus ou moins calquée sur celle de pays comme la France à la même période et se donne pour objet de remettre en état les édifices pénitentiaires, d'améliorer l'administration et la surveillance dans les établissements, d'y organiser le travail, d'en améliorer le service économique et, enfin, d'organiser la déportation et les travaux forcés ainsi que de développer le service des transferts.

14. Voir par exemple le témoignage d'un membre éminent de la Ligue des Droits de l'Homme (LDH) : Pressensé F. (de), Atrocities in Russian prisons, translated from the French [biographical sketch of Francis de Pressensé, by E. Roubanovitch], London, Society of friends of Russian Freedom, 1913. Sur la politique pénitentiaire en Russie avant la Révolution de 1917 voir l'ouvrage important d'Adams B., The politics of punishment : prison reform in Russia, 1863-1917, Dekalb, Nathermn Illinois University press, 1996.

15. Après la Révolution, en 1928, elle devient un musée puis est détruite par les Allemands pendant la Seconde Guerre mondiale.

16. Notre intérêt pour le cas de Véra Figner n'est pas sans lien avec l'histoire contemporaine française. Au début des années 1970, naît à l'initiative de Michel Foucault notamment, le Groupe d'Information sur les Prisons (GIP). L'une des spécificités de cette lutte fut de s'appuyer sur les témoignages de prisonniers (voir la contribution de G. Salle dans le présent numéro). Le GIP se donna pour tâche de "faire-savoir » la prison à partir des savoirs individuels des détenus. Le groupe développa ainsi une enquête dans les prisons françaises à l'aide d'un questionnaire mais il encouragea aussi la production de récits autobiographiques dont certains furent publiés. La brochure Intolérable sur le suicide en prison en 1972 fut ainsi l'occasion de la publication de lettres d'un détenu (Artières Ph., Quéro L., Zancarini M., Le Groupe d'Information sur les Prisons. Archives d'une lutte. 1970-1972, Paris, Ed. de l'Institut Mémoires de l'édition contemporaine, 2003). Cet usage militant de l'autobiographie de prisonniers qui s'inscrit dans ce que Michel de Certeau désigna comme la prise de parole (Certeau M., La prise de parole : pour une nouvelle culture, Paris, éd. Desclée de Brouwer, 1968 ; Artières Ph., "La prison en procès : les mutins de Nancy (1972) », Vingtième Siècle, ${ }^{\circ} 70,2001$, pp.57-70.) a une archéologie. Les luttes que menèrent au cours des années 1870-1917, les révolutionnaires russes sont, comme on le verra, le théâtre d'une résistance aux prisons et aux bagnes russes par le témoignage personnel. Le cas de Véra Figner et des populistes russes de la «Volonté du Peuple» est particulièrement exemplaire de ces techniques de résistance par l'écriture autobiographique.

17. RGALI : Cote 1185 op. 1. 146. Un autre fonds Figner qui regroupe la correspondance n'a pu être consulté aux archives centrales de Moscou. Certaines archives Figner sont également conservées à la Bibliothèque de Documentation Internationale Contemporaine (BDIC-Université de Paris 10 Nanterre) dans le fonds Eugène Petit (correspondances, cartes postales, photos et cartons illustrés : F delta Rés 571 (5)-3).

18. Pour cette enquête ont été en particulier utilisés: Dictionnaire encyclopédique de l'Institut bibliographique russe,Granat, Septième et huitième livraisons du tome 40, Socialisme: Annexe à l'article «Le développement de la pensée socialiste en Russie», Autobiographies de révolutionnaires du mouvement socialiste russe des années 1870 et de la première moitié des 
années 1880. Notes de V. Figner : 44 autobiographies, écrites en 1925-1926 (p. 1-658); Wolkenstein L., 13 années dans la forteresse de Schlüsselbourg, éd. A. Tchertkoff, 1900 ; Novorousski M., Notes d'un Schlüsselbourgeois. 1887-1905, Pétersbourg, 1920 ; La revue Byloïé [revue consacrée à l'histoire du mouvement de libération] dont: Aschenbrenner M., "La prison de Schlüsselbourg durant 20 ans, de 1884 à 1904. Souvenirs de M. A. », Byloïé, n¹, janvier 1906, pp. 54-93; Pankratov V., «En hommage à L. Wolkenstein ", Byloié, n², février 1906, pp. 280-282 ; Figner V., "La prisonnière de Schlüsselbourg L. Wolkenstein », Byloié, n³, mars 1906, pp. 253-275; Société des amis du peuple russe et des peuples annexes, publications périodiques de la Société des amis du peuple russe et des peuples annexes, 1905-1909 (fascicules n¹-16) ; Petit E., Une Bastille russe, la forteresse de Sclüsselbourg, conférence faite a paris le 30 juin 1906, Paris, Société des amis du peuple russe et des peuples annexes; Kropotkin P.A., La terreur en Russie (un appel a la nation britannique), publié par le comité parlementaire russe, Paris, Stock, 1910.; Kropotkin P.A, In the Russian and French prisons, New-York, Shodken books, 1971.

19. Aschenbrenner M., «La prison de Schlüsselbourg durant 20 ans, de 1884 à 1904. Souvenirs de M. A. ", op. cit., p. 71.

20. Dans une autre lettre à sa mère, datée de 2 mars 1897 et publiée dans les Euvres complètes de V. Figner (tome 6, Lettres, Moscou, 1932, p. 175), on retrouve cette impuissance à écrire: "Chère petite maman, Il y a deux semaines, le 16 février, j'ai reçu votre lettre du 13, et on m'a dit que je pouvais vous écrire deux fois par an... La correspondance est pour moi une affaire si extraordinaire que, étant parfois par la pensée des journées entières avec vous tous et m'entretenant librement dans mon sommeil et à l'état de veille avec vous, je suis complètement perdue, en m'attaquant au papier... ».

21. Dans ses mémoires, Figner raconte sa sortie de détention et rapporte qu'en attendant le bateau qui doit l'emmener à Pétersbourg, le commandant lui demande : «Ne voulez-vous pas du thé, Véra Nicolaievna ?». Celle-ci de commenter : «Comme est bon ce 'Véra Nicolaïevna' ! Durant vingt ans, il n'y avait pas eu pour moi de nom 'chez eux'. vingt années, j'avais été pour eux seulement un numéro : 'le n'11' c'est ainsi qu'ils m'appelaient constamment ; il y a dix minutes, j'étais encore le $n^{\circ} 11 .$. et maintenant tout à coupj'étais devenue Véra Nicolaïevna ».

22. Dans cet ouvrage, l'ancienne co-détenue de Véra Figner précisait le sens de son entreprise autobiographique: "Décrire en détail une vie étendue sur une si longue période, une vie dans laquelle furent éprouvées tellement de choses intérieurement et si peu de l'extérieur est très difficile. Il serait possible de le faire seulement avec un état d'esprit qui convienne parfaitement... En tout cas, ces esquisses peuvent donner une certaine idée de la prison de Schlüsselbourg et peuvent servir de matériau à un futur historien de celle-ci... ", Wolkenstein L., op. cit., p. 5.

23. Extrait de la brochure que Figner rédige vers 1910 en Suisse intitulée : " Les Prisons russes ».

24. Dans la version publiée à Moscou en 1932, Véra Figner indique dans le Chapitre LIII intitulé «La Suisse »: "C'est la Suisse qui était mon lieu de séjour permanent... Je me produisais à des moments divers à Zurich : une fois, à une soirée étudiante où le célèbre social-démocrate Grélikh prononça un brillant discours sur Schlüsselbourg, je récitai mon poème 'Le bonheur nous est échu', mis en musique par un étudiant... Une autre fois, je lus au profit des affamés l'article qui n'avait pas encore été imprimé 'Avec une poignée d'or'. C'est dans le même but que je lus ce chapitre à Zurich, Lausanne, à Genève et à Leysin... A Genève fut organisé un meeting en français très réussi, réunissant beaucoup de gens... A Lausanne, je fis un exposé... formation d'un comité autonome russe qui... se chargea de prendre soin d'un des bagnes... Mon initiative... inspira celle des colonies russes également dans des villes où je n'apparaissais pas personnellement : à Grenoble, Toulouse, à Nancy, se formèrent des sections du Comité parisien ou des comités autonomes... ». 


\section{RÉSUMÉS}

Emprisonnée pendant vingt ans à la forteresse de Schusselbourg, pour sa participation au mouvement La volonté du peuple, responsable de plusieurs attentats dont l'assassinat du tsar Alexandre II, la populiste russe Vera Figner mène d'abord en détention puis dès sa sortie en 1906 une entreprise singulière de résistance par l'écriture. La Venus de la Révolution entreprend en effet de constituer son récit autobiographique d'emprisonnement en un outil de lutte contre l'arbitraire du pouvoir tsariste. Si, avant 1917, il s'agit pour elle de constituer la longue et douloureuse incarcération d'elle et de ses camarades en un symbole de la répression, la rédaction de ses mémoires après la Révolution participe de l'écriture d'une histoire des prisons et des mouvements de résistance qui s'y développèrent.

The Russian populist Vera Figner, responsible of several attacks among which the murder of Tzar Alexander II was imprisoned during twenty years in the Schlusselburg fortress for her participation in the People's Will movement. During her time in prison, and then later in 1906 when she was freed, she undertook the peculiar action of resistance through writing. The Revolution's Venus indeed chose to make her autobiographical detention story a weapon against the tsarist power arbitrary. If prior to 1917 this meant constituting the long and painful incarceration she and her comrades went through into a symbol of repression, the writing of her memories after the Revolution participated in writing the story of prisons and of resistance movements within them.

\section{INDEX}

Mots-clés : Vera Figner, prison, résistances politiques, écriture, russe

\section{AUTEURS}

\section{PHILIPPE ARTIÈRES}

Philippe Artières est chargé de recherche au CNRS (LAHIC-UMR2558). Il a publié avec Pierre Lascoumes (dir.), Gouverner, enfermer. La prison, un modèle indépassable? (Paris, Presses de Sciences Po, 2004).

\section{DENIS DABBADIE}

Denis Dabbadie est professeur agrégé de russe et membre de l'équipe genèse \& autobiographie ITEM/CNRS. 